Check for updates

Cite this: Phys. Chem. Chem. Phys., $2018,20,22463$

Received 13th July 2018 Accepted 14th August 2018 DOI: $10.1039 / \mathrm{c} 8 \mathrm{cp} 04448 a$ rsc.li/pccp

\section{Nuclear spin singlet states as magnetic on/off probes in self-assembling systems}

\author{
Salvatore Mamone ${ }^{a b}$ and Stefan Glöggler iD *ab
}

Self-assembling processes occur in a variety of compounds such as peptides, proteins and DNA. These processes have been linked to pathologies and have as well been exploited for designing responsive contrast agents for disease detection. Novel methods to investigate and detect self-assembly therefore hold promise to obtain more insights into disease progression or open pathways to the design of novel self-assembling materials. In this article we are introducing nuclear singlet states to probe self-assembly in the dipeptide isoleucine-phenylalanine (IF) as a thermoresponsive on/off switch for nuclear magnetic resonance (NMR). We have investigated the relaxation and singlet state properties of the $\beta$-protons of phenylalanine in the IF dipeptide in aqueous solutions. At IF concentrations of $2 \mathrm{wt} \%$ and above $308 \mathrm{~K}$, a long lived nuclear singlet state, as compared to the longitudinal relaxation, was observed. At $308 \mathrm{~K}$ the dipeptide starts forming a gel and no singlet state is accessible at lower temperatures. Upon heating, the gel disassembles and an isotropic liquid forms making the singlet state accessible again. This demonstrates the thermoresponsive on-off character of the nuclear spin singlet state in the IF dipeptide.

\section{Introduction}

In a self-assembly process, various individual components arrange themselves into an ordered structure. ${ }^{1}$ Examples of materials that self-assemble include DNA, peptides, proteins, nanocrystals and nanoparticles. ${ }^{1-27}$ Triggered self-assembly of nanostructures or disassembly of these materials has been investigated in recent years for the design of magnetic resonance imaging (MRI) contrast agents. ${ }^{18-27}$ In particular, labelling selfassembling molecules with ${ }^{19} \mathrm{~F}$ nuclei has moved into the focus. $^{23-27}$ Thereby, two concepts have been pursued. ${ }^{26,27}$ Firstly, hydrophilic molecules that have a reactive species are attached to a hydrophobic group containing fluorine and selfassemble into a nanostructure with the fluorine nuclei in the core. Interaction of the reactive group with $e . g$. an enzyme leads to disassembly of the nanostructure. Secondly, the reactive group in the described molecules is blocked but gets revealed and regains its reactivity upon a stimulus, subsequently leading to a self-assembled structure. Both processes can be observed via ${ }^{19}$ F-NMR. ${ }^{26,27}$ If the molecule is in its disassembled state, short correlation times and isotropic molecular motion lead to a narrow observable peak in the ${ }^{19} \mathrm{~F}$-NMR spectrum. Once selfassembled, the ${ }^{19} \mathrm{~F}$-nuclei (in the core of the particle) are restricted in their motion and experience increased dipolar interactions and

\footnotetext{
${ }^{a}$ Max Planck Institute for Biophysical Chemistry, Am Faßberg 11, 37077 Göttingen, Germany.E-mail: stefan.gloeggler@mpibpc.mpg.de

${ }^{b}$ Center for Biostructural Imaging of Neurodegeneration of $U M G$,

Von-Siebold-Straße 3A, 37075 Göttingen, Germany
}

chemical shift anisotropy (CSA), leading to a broadening of the observable peak until it is not detectable anymore. In other words, the spin-spin relaxation time $T_{2}$ becomes shorter in the selfassembled structure to the point that the NMR signal is not detectable anymore, hence resulting in an on/off switch depending on the assembly state. As an alternative that does not require labelling with ${ }^{19} \mathrm{~F}$, we have explored on/off-switches for proton nuclear singlet states. Singlet states are nuclear spin states with effective spin 0 that can be formed between a pair of spin $1 / 2$ nuclei which are dynamically isolated from the rest of the spin system. ${ }^{28-61}$ Interestingly, singlet states are immune to the direct dipole-dipole relaxation between the constituent spins in fast and isotropically tumbling molecules, which is often the main relaxation mechanism for the longitudinal and transverse magnetization modes. As such, singlet states are often characterized by singlet-triplet equilibration times $T_{\mathrm{s}}$ that exceed the spin-lattice relaxation times $T_{1}$. Although the longest singlet lifetimes (over 1 hour), ${ }^{44}$ were found between spin pairs close to magnetic equivalence in strongly coupled systems, singlet states can be generated for systems in which the nuclei are far from magnetic equivalence and weakly coupled, ${ }^{28}$ for example in $\alpha$-protons of glycine in small peptides and $\beta$-protons in amino acids and peptides. $^{33,34,38}$ This observation has e.g. been explored in the development of a detection method for binding affinity, which has proven to be more sensitive than $T_{1}$-based methods. ${ }^{38}$

In this work we have explored the feasibility of utilizing nuclear singlet states as on/off switches and demonstrate that such a behaviour can be observed in $\beta$-protons of the dipeptide isoleucine-phenylalanine (IF), which is shown in Fig. 1 together 
A)

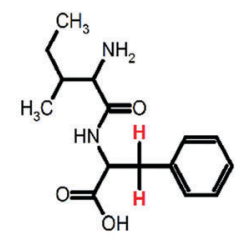

Isoleucine-Phenylalanine
B)

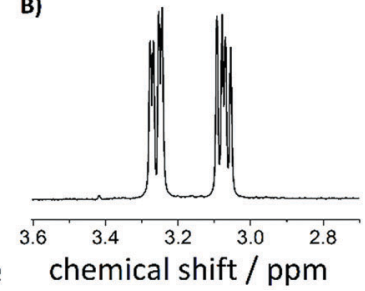

Fig. 1 (A) IF dipeptide (B) ${ }^{1} \mathrm{H}$ NMR spectrum of the two beta protons of $F$.

with the respective NMR spectrum of the two protons of interest. For investigating the nuclear singlet state in $\mathrm{H}_{2} \mathrm{O}$, we used a singlet filter sequence with water suppression. The sequence can be applied to weakly coupled spin systems (as it is the case here) and includes an extension of the previously published $\mathrm{T}_{00}$-filter. ${ }^{61}$ We show that the dipeptide acts as a thermoresponsive switch for singlet states, which can be populated (turned on) at a temperature of $308 \mathrm{~K}$ and are not accessible below this temperature (turned off). This is due to the fact that below the critical temperature a hydrogel ${ }^{62}$ is formed in which the correlation time is significantly increased. Our investigations on this molecule and its behaviour are presented in the following.

\section{Experimental section}

The dipeptide isoleucine-phenylalanine was purchased from Bachem (product number 4001668.0001) and used without further purification. Two degassed NMR samples were prepared with either $0.5 \mathrm{wt} \%$ or $2 \mathrm{wt} \%$ dipeptide with respect to a mixture of $95 \%$ deionized $\mathrm{H}_{2} \mathrm{O}$ and $5 \% \mathrm{D}_{2} \mathrm{O}$. NMR experiments were performed on a AV600 HDIII Bruker system with variable temperature setup at $600 \mathrm{MHz}$ proton frequency (corresponds to $B_{0}=14.1 \mathrm{~T}$ ). To estimate the longitudinal relaxation times $T_{1}$, an inversion recovery experiment was performed at different temperatures from $323 \mathrm{~K}$ to $283 \mathrm{~K}$. $T_{\mathrm{S}}$ was measured at the same temperatures with the recently introduced APSOC sequence including an extra PE-WATERGATE ${ }^{63}$ block for water suppression in the ${ }^{1} \mathrm{H}$ NMR spectrum. Furthermore, we have added a singlet filter $\left(\mathrm{T}_{00}\right)$ that can be utilized for weakly coupled spin systems. The whole sequence is shown in Fig. 2 with the respective timings. Compared to the $\mathrm{T}_{00}$ filter reported earlier, ${ }^{61}$ three 180 degree pulses have been added in order to remove the effect of offset evolution, and timings $\tau_{\mathrm{r}}$ close to $\tau^{*}=\left[J^{2}+\Delta \nu^{2}\right]^{-0.5}$, which corresponds to a full rotation in the zero-quantum space, have been used. Here $J$ and $\Delta \nu$ represent the $J$-coupling and the chemical shift difference between the two nuclei measured in $\mathrm{Hz}$, respectively. Further details of the $\mathrm{T}_{00}$ filter will be published elsewhere. Spinspin relaxation times $T_{2}$ were measured utilizing a CPMG sequence for both samples. CPMG echo times were $0.4 \mathrm{~ms}$ for the $0.5 \mathrm{wt} \%$ sample and for the $2 \mathrm{wt} \%$ sample above $308 \mathrm{~K}$ and $0.2 \mathrm{~ms}$ for the 2 wt\% sample below $308 \mathrm{~K}$.

\section{Results and discussion}

\subsection{Evaluation of the singlet filter}

For the investigation of nuclear singlet states in a weakly coupled spin system (as it is the case in the IF peptide), we

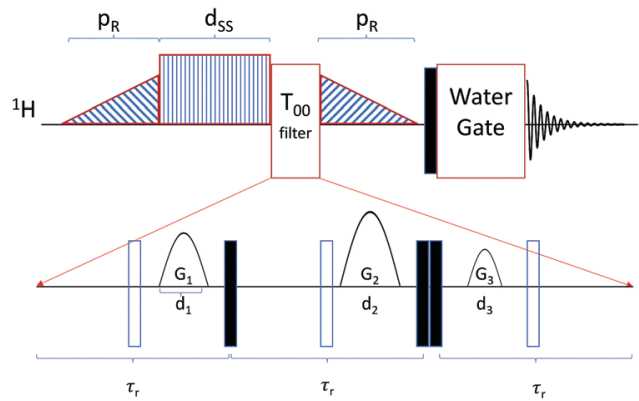

Fig. 2 Schematic of the APSOC sequence used in this work. The filled and empty rectangles represent $\pi / 2$ and $\pi$ pulses, respectively. The sequence consists of a ramped RF passages with duration of $p_{R}=200 \mathrm{~ms}$ and maximum RF field corresponding to a nutation frequency of $600 \mathrm{~Hz}$ which converts $z$-magnetization into the singlet state. The offset of the singlet excitation and reconversion ramps was $\pm 23 \mathrm{~Hz}$ with respect to the resonances of the beta protons of the phenylalanine residue. The singlet sustaining block consists of a CW RF field with nutation frequency $1 \mathrm{kHz}$ in order to quench chemical shifts induced oscillations in the singlet state which is turned on for the duration $d_{\mathrm{Ss}}$. The $T_{00}$ filter greatly reduces any signal orthogonal to the singlet state before it is converted back to $z$-magnetization by the ramped down RF passage. The acquisition block consists of a hard $\pi / 2$ pulse, with nutation frequency of $25 \mathrm{kHz}$, followed by a PE-WATERGATE. ${ }^{63}$ In the $T_{00}$ block, the duration $\tau_{\mathrm{r}}$ of the spin echoes were $8 \mathrm{~ms}$, the durations of the gradients $d_{1}=d_{2}=d_{3}=2 \mathrm{~ms}$ and the gradients were $\left\{G_{1}, G_{2}, G_{3}\right\}=\{2,27.5,7\}$ in $\mathrm{G} \mathrm{cm}^{-1}$.

introduce a filter that is offset independent. The rational for the development is based on the idea that typically proton background signals occur in biological environments. Regarding the background signals, water is the most dominant, which is depicted in Fig. 3A. The concentration of water $(\approx 55 \mathrm{M})$ often exceeds physiological metabolite concentrations by more than 10000 -fold. ${ }^{64}$ This may lead to masking of the desired signal to be observed. In order to increase the detectability from lowconcentration component, water suppression techniques have been introduced for in vitro an in vivo experiments. ${ }^{63,65-67}$ To demonstrate the effect of a water suppression sequence on the investigated system we have performed a PE-WATERGATE experiment, that reduces the water signal by more than 1000 -fold and the result is shown in Fig. 3B. ${ }^{63}$ From the spectrum it becomes evident that the water signal has significantly been suppressed but other ${ }^{1} \mathrm{H}$ signals from the dipeptide are still observable. Under physiological conditions in e.g. cells or in vivo many more ${ }^{1} \mathrm{H}$ signals may be present that may mask the signal of interest. In order to remove them we introduced a $\mathrm{T}_{00}$-filter for weakly coupled spin systems (see Fig. 2) that, in combination with WATERGATE, suppresses other proton signals than the desired one and also manages to suppress the water signal even further. The result is shown in Fig. 3C. This follows an idea presented in ref. 41 with the advantage that the $\mathrm{T}_{00}$-filter presented here is offset independent and may be combined with offset independent singlet NMR sequences such as the M2S-S2M sequence for imaging purposes. ${ }^{32}$

\subsection{Investigation of the dipeptide isoleucine-phenylalanine (IF)}

One of the IF properties includes the reversible gelling upon temperature changes ${ }^{62}$ above a critical concentration. If an IF sample with $2 \mathrm{wt} \%$ in water is investigated the peptide starts 
A)
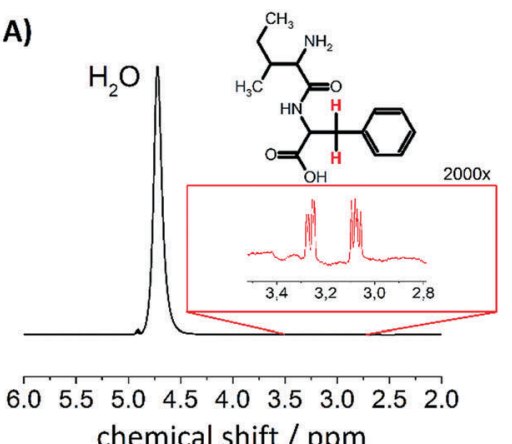

B)

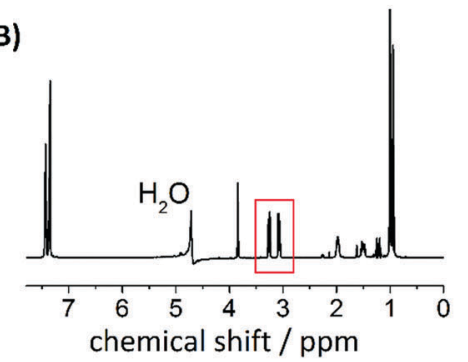

C)

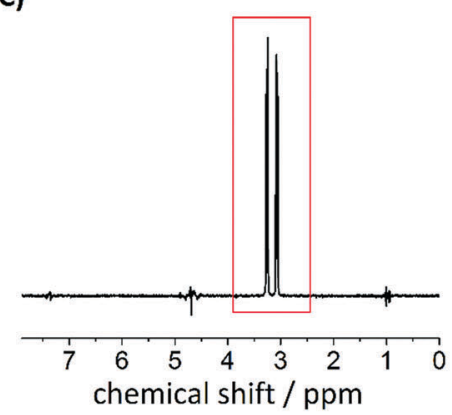

Fig. 3 Effect of singlet filter. (A) Single scan ${ }^{1} \mathrm{H}$ NMR spectrum of $0.5 \mathrm{wt} \%$ (16 mM) IF dipeptide dissolved in $95 \% \mathrm{H}_{2} \mathrm{O}$ and $5 \% \mathrm{D}_{2} \mathrm{O}\left(B_{0}=14.1 \mathrm{~T}\right)$. The inset shows the proton signal of the two indicated protons with 2000 -fold magnification. (B) ${ }^{1} \mathrm{H}$ NMR WATERGATE spectrum of the same sample (8 scans). (C) ${ }^{1} \mathrm{H}$ NMR after applying the singlet filter for weakly coupled spin systems with $d_{\mathrm{ss}}=200 \mathrm{~ms}$ of singlet sustaining duration after the first APSOC ramp (32 scans).

forming a gel at $303 \mathrm{~K}$. This process can be monitored with NMR by observing a line broadening that occurs around this temperature. ${ }^{62}$ Fig. $4(\mathrm{~A})$ shows the spectra at $313 \mathrm{~K}$ at which the dipeptide is in its monomeric form with isotropic movement leading to narrow lines and after the gel has formed at $293 \mathrm{~K}$ in $4 \mathrm{~B}$ with broadened lines. In case of a $0.5 \mathrm{wt} \%$ sample, no gelification occurs and the dipeptides keep up the isotropic movement.

We have investigated the longitudinal and transverse ${ }^{1} \mathrm{H}$ relaxation properties as well as the singlet lifetime $T_{\mathrm{S}}$ as a function of temperature in a $\mathrm{H}_{2} \mathrm{O}: \mathrm{D}_{2} \mathrm{O}$ mixture $(95 \%: 5 \%)$ for the $0.5 \mathrm{wt} \%$ and $2 \mathrm{wt} \%$ dipeptide. The results are displayed in Fig. 5 . For the $0.5 \mathrm{wt} \%$ sample, $T_{\mathrm{S}}$ remains about three to four times longer than $T_{1}$ over a temperature range from $313 \mathrm{~K}$ to $283 \mathrm{~K}$. Considering that relaxation of protons in peptides is usually dominated by intramolecular dipolar relaxation, we used the ${ }^{1} \mathrm{H}$ transverse relaxation times to estimate a correlation a)

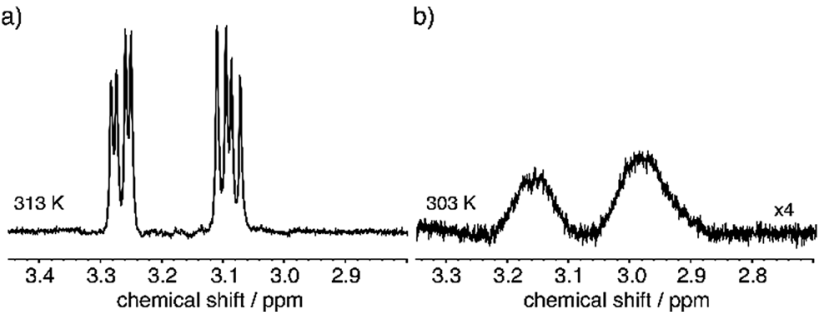

Fig. 4 Comparison of the phenylalanine beta protons at (a) $313 \mathrm{~K}$ and (b) $303 \mathrm{~K}$. Formation of the gel becomes evident by the observed line broadening.

time $\tau_{\mathrm{c}(\mathrm{DD})}{ }^{68}$ according to the following formula previously used for the investigation in stretched hydrogels ${ }^{69}$ whereby $\omega_{0}$ represents the angular Larmor frequency:

$$
\begin{aligned}
& \tau_{\mathrm{c}(\mathrm{DD})} \\
& =\frac{1}{2 \sqrt{6 \omega_{0}}} \sqrt{-37+16\left(\frac{T_{1}}{T_{2}}\right)+\sqrt{889-704\left(\frac{T_{1}}{T_{2}}\right)+256\left(\frac{T_{1}^{2}}{T_{2}^{2}}\right)}}
\end{aligned}
$$

The equation was shown to be valid in regions where $\tau_{\mathrm{c}(\mathrm{DD})}<10 \mathrm{~ns}$ and is applicable if intramolecular dipolar interactions can be assumed the most dominant relaxation mechanism. ${ }^{69}$ At $313 \mathrm{~K}$ we estimate a correlation time of $92 \mathrm{ps}$ and at $303 \mathrm{~K}$ of $94 \mathrm{ps}$ for the $0.5 \mathrm{wt} \%$ sample in which the molecules undergoes isotropic motion at all times.

Upon investigation of the $2 \mathrm{wt} \%$ dipeptide at the same temperatures, it becomes evident that the relaxation times as well as the singlet lifetime behave similarly until the transition into a gel occurs. At $308 \mathrm{~K}$ we observed an intermediate state in which the singlet state can be still accessed is however almost on the order of $T_{1}\left(T_{1}=520 \mathrm{~ms}\right.$ and $\left.T_{\mathrm{S}}=700 \mathrm{~ms}\right)$. Below $303 \mathrm{~K}$ the singlet state is not accessible anymore. Investigations of the transverse relaxation time $T_{2}$ show a drop from $400 \mathrm{~ms}$ at $313 \mathrm{~K}$ to $5.4 \mathrm{~ms}$ at $303 \mathrm{~K}$. $T_{1}$ is reduced from $570 \mathrm{~ms}$ to $250 \mathrm{~ms}$. Using eqn (1) we estimate a $\tau_{\mathrm{c}(\mathrm{DD})}$ of $132 \mathrm{ps}$ at $313 \mathrm{~K}$ and $2.04 \mathrm{~ns}$ at $303 \mathrm{~K}$. In the gel case, the molecular motion does not fall into the extreme narrowing limit anymore $\left(\omega_{0} \tau_{c} \ll 1\right)$, for which most of the relaxation properties of the singlet states have been investigated so far. The utilized equation however was shown to be applicable for a gel in the past and supports the measured $T_{\mathrm{S}}$ data. ${ }^{69}$ As $T_{\mathrm{S}}$ is inverse proportional to the correlation time, ${ }^{70}$ we regard the increase in correlation time as the main reason a)

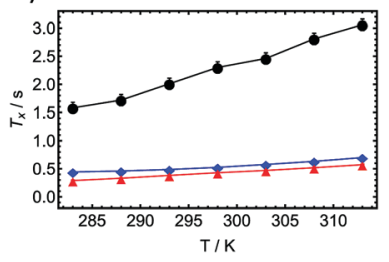

b)

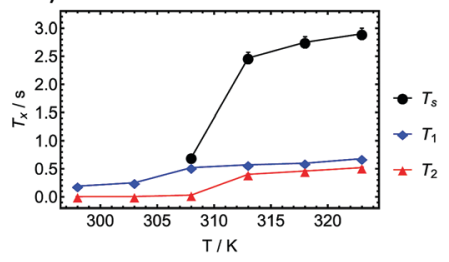

Fig. $5 T_{\mathrm{s}}, T_{1}$ and $T_{2}$ relaxation times of the beta protons in the $\mathrm{F}$ residue of the dipeptide IF, as a function of temperature for (a) $0.5 \mathrm{wt} \%$ and (b) $2 \mathrm{wt} \%$. Below $308 \mathrm{~K}$ no singlet state was accessible in the $2 \mathrm{wt} \%$ sample. 
for the reduction in the time for singlet-triplet equilibration. The ratio of the correlation times of the liquid state to the gel state amounts to 15.4 . If $T_{\mathrm{S}}$ is reduced by that factor in the gel we expect a drop from the measured $T_{\mathrm{S}}(313 \mathrm{~K})=2470 \mathrm{~ms}$ to $T_{\mathrm{S}}(303 \mathrm{~K})=160 \mathrm{~ms}$. Since the sequence timings (two $200 \mathrm{~ms}$ APSOC ramps, sustaining period and filter) exceed three times the estimated $T_{\mathrm{S}}$ at $303 \mathrm{~K}$, relaxation effects may impede the effective observation of the singlet state.

The observed behaviour in the gels is completely reversible i.e. the detectable singlet-states can be switched "on" or "off", by controlling the temperature. The accessibility of the singlet state after its back conversion from the gel holds therefore promise to develop stimuli responsive contrast agents with an on/off switch. This mechanism is not based on line broadening effects but utilizes the storage of magnetization in proton singlet states in combination with an efficient filter to suppress background signals.

\section{Conclusions}

In conclusion, we have introduced an extension of the $\mathrm{T}_{00}$ filter that can be utilized for weakly coupled spin systems. In combination with water suppression techniques, proton signals other than the desired ones are effectively removed in the context of singlet NMR. Additionally, we have demonstrated the feasibility of a stimuli-responsive on/off switch for nuclear singlet states based on a thermo-responsive gel formed from the isoleucine-phenylalanine dipeptide. Overall, our investigations demonstrate the possibility of switchable contrast agents that utilize nuclear singlet states of protons without the need of isotopic labelling.

\section{Conflicts of interest}

The authors declare no conflict of interest.

\section{Acknowledgements}

The authors would like to acknowledge generous funding from the Max-Planck-Society and Prof. Christian Griesinger for access to his equipment and facilities. Open Access funding provided by the Max Planck Society.

\section{Notes and references}

1 M. A. Boles, M. Engel and D. V. Talapin, Chem. Rev., 2016, 116, 11220.

2 L. Sun, C. Zheng and T. J. Webster, Int. J. Nanomed., 2017, 12, 73. 3 T. Fan, X. Yu, B. Shen and L. Sun, J. Nanomater., 2017, 4562474.

4 A. M. Nyström, J. W. Bartels, W. Du and K. L. Wooley, J. Polym. Sci., Part A: Polym. Chem., 2009, 47, 1023.

5 M. Grzelczak, J. Verman, E. M. Furst and L. M. Liz-Marzan, ACS Nano, 2010, 4, 3591.

6 J. J. McManus, P. Charbonneau, E. Zaccarelli and N. Asherie, Curr. Opin. Colloid Interface Sci., 2016, 22, 73.
7 Y. Bai, Q. Luo and J. Liu, Chem. Soc. Rev., 2016, 45, 2756.

8 R. Faas, A. Pohle, K. Moß, M. Henkel and R. Hausmann, Biotechnol. Rep., 2017, 16, 1.

9 H. Li, J. D. Cater and T. H. LaBean, Mater. Today, 2009, $12,24$.

10 G. Whitsides and B. Grzybowski, Science, 2002, 295, 2418.

11 L. L. Ong, N. Hanikel, O. K. Yaghi, C. Grun, M. T. Strauss, P. Bron, J. Lai-Kee-Him, F. Schueder, B. Wang, P. Wang, J. Y. Kishi, C. Myhrvold, A. Zhu, R. Jungmann, G. Bellot, Y. Ke and P. Yin, Nature, 2017, 552, 72.

12 P. W. K. Rothemund, Nature, 2006, 440, 297.

13 E. Winfree, F. Liu, L. A. Wenzler and N. C. Seeman, Nature, 1998, 394, 539.

14 Y. Taniguchi, M. A. Bin Sazali, Y. Kobayashi, N. Arai, T. Kawai and T. Nakshima, ACS Nano, 2017, 11, 9312.

15 D. Vanmaekelbergh, Nano Today, 2011, 6, 419.

16 Y.-C. Lin, C.-Y. Chen, H.-L. Chem, T. Hashimoto, S.-A. Chen and Y.-C. Li, J. Chem. Phys., 2015, 142, 214905.

17 N. Li, A. Z. Panagiotopoulos and A. Nikoubashman, Langmuir, 2017, 33, 6021.

18 C. Frangville, Y. Li, C. Billotey, D. R. Talham, J. Taleb, P. Roux, J.-D. Marty and C. Mingotaud, Nano Lett., 2016, 16, 4069.

19 G. Liang, J. Ronald, Y. Chen, D. Ye, P. Pandit, M. L. Ma, B. Rutt and J. Rao, Angew. Chem., Int. Ed., 2011, 50, 6283.

20 Z. Zheng, H. Sun, C. Hu, G. Li, X. Liu, P. Chen, Y. Cui, J. Liu, J. Wang and G. Liang, Anal. Chem., 2016, 88, 3363.

21 S. Bo, C. Song, Y. Li, W. Yu, S. Chen, X. Zhou, Z. Yang, X. Zheng and Z.-X. Jiang, J. Org. Chem., 2015, 80, 6360.

22 A. T. Preslar, T. Tantakitti, K. Park, S. Zhang, S. I. Stupp and T. J. Meade, ACS Nano, 2016, 10, 7376.

23 M. Buzhor, L. Avram, L. Frish, Y. Cohen and R. J. Amir, J. Mater. Chem. B, 2016, 4, 3072.

24 X. Huang, G. Huang, S. Zhang, K. Sagiyama, O. Togao, X. Ma, Y. Wang, Y. Li, T. C. Soesbe, B. D. Sumer, M. Takahashi, A. D. Sherry and J. Gao, Angew. Chem., Int. Ed., 2013, 52, 8074.

25 K. Matsuo, R. Kamada, K. Mizusawa, H. Imai, Y. Takayama, M. Narazaki, T. Matsuda, Y. Takaoka and I. Hamachi, Chem. Eur. J., 2013, 19, 12875.

26 Y. Takaoka, T. Sakamoto, S. Tsukiji, M. Narazaki, T. Matsuda, H. Tochio, M. Shirakawa and I. Hamachi, Nat. Chem., 2009, 1, 557.

27 Y. Yuan, S. Ge, H. Sun, X. Dong, H. Zhao, L. An, J. Zhang, J. Wang, B. Hu and G. Liang, ACS Nano, 2015, 9, 5117.

28 M. Carravetta and M. H. Levitt, J. Am. Chem. Soc., 2004, 126, 6228-6229.

29 M. Carravetta, O. G. Johannessen and M. H. Levitt, Phys. Rev. Lett., 2004, 92, 153003.

30 M. Carravetta and M. H. Levitt, J. Chem. Phys., 2005, 122, 214505. 31 G. Pileio and M. H. Levitt, J. Chem. Phys., 2009, 130, 214501.

32 G. Pileio, M. Carravetta and M. H. Levitt, Proc. Natl. Acad. Sci. U. S. A., 2011, 107, 17135-17139.

33 M. C. D. Tayler, S. Marie, A. Ganesan and M. H. Levitt, J. Am. Chem. Soc., 2010, 132, 8225.

34 A. N. Pravdivtsev, A. V. Yurkovskaya, H. Zimmermann, H.-M. Vieth and K. L. Ivanov, Phys. Chem. Chem. Phys., 2014, 16, 7584 . 
35 R. Sarkar, P. R. Vasos and G. Bodenhausen, J. Am. Chem. Soc., 2007, 129, 328-334.

36 P. Ahuja, R. Sarkar, P. R. Vasos and G. Bodenhausen, J. Chem. Phys., 2007, 127, 134112.

37 R. Buratto, D. Mammoli, E. Chiarparin, G. Williams and G. Bodenhausen, Angew. Chem., Int. Ed., 2014, 53, 11376-11380.

38 N. Salvi, R. Buratto, A. Bornet, S. Ulzega, I. R. Rebollo, A. Angelini, C. Heinis and G. Bodenhausen, J. Am. Chem. Soc., 2012, 134, 11076-11079.

39 R. Buratto, A. Bornet, J. Milani, D. Mammoli, B. Vuichoud, N. Salvi, M. Singh, A. Laguerre, S. Passemard, S. GerberLemaire, S. Jannin and G. Bodenhausen, ChemMedChem, 2014, 9, 2509-2515.

40 R. Buratto, D. Mammoli, E. Canet and G. Bodenhausen, J. Med. Chem., 2016, 59, 1960-1966.

41 A. S. Kiryutin, A. N. Pravdivtsev, A. V. Yurkovskaya, H.-M. Vieth and K. L. Ivanov, J. Phys. Chem. B, 2016, 120, 11978-11986.

42 G. Pileio, M. Carravetta, E. Hughes and M. H. Levitt, J. Am. Chem. Soc., 2008, 130, 12582-12583.

43 M. C. D. Tayler and M. H. Levitt, Phys. Chem. Chem. Phys., 2011, 13, 5556-5560.

44 G. Pileio, M. Concistre, M. Carravetta and M. H. Levitt, J. Magn. Reson., 2006, 182, 353-357.

45 T. Theis, Y. Feng, T. Wu and W. S. Warren, J. Chem. Phys., 2014, 140, 014201.

46 G. Stevanato, J. T. Hill-Cousins, P. Håkansson, S. S. Roy, L. J. Brown, R. C. D. Brown, G. Pileio and M. H. Levitt, Angew. Chem., Int. Ed., 2015, 54, 3740-3743.

47 S. J. Elliott, L. J. Brown, J.-N. Dumez and M. H. Levitt, Phys. Chem. Chem. Phys., 2016, 18, 17965-17972.

48 Y. Feng, R. M. Davis and W. S. Warren, Nat. Phys., 2012, 8, 831-837.

49 A. K. Grant and E. Vinogradov, J. Magn. Reson., 2008, 194, 46-57.

50 W. S. Warren, E. Jensita, R. T. Branca and X. Chen, Science, 2009, 323, 1711-1714.

51 G. Pileio, S. Bowen, C. Laustsen, M. C. D. Tayler, J. T. HillCousins, L. J. Brown, R. C. D. Brown, J. H. Ardenkjaer-Larsen and M. H. Levitt, J. Am. Chem. Soc., 2013, 135, 5084-5088.
52 Y. Feng, T. Theis, X. Liang, Q. Wang and W. S. Warren, J. Am. Chem. Soc., 2013, 135, 9632-9635.

53 T. Theis, G. X. Ortiz Jr., A. W. J. Logan, K. E. Claytor, Y. Feng, W. P. Huhn, V. Blum, S. J. Malcolmson, E. Y. Chekmenev, Q. Wang and W. S. Warren, Sci. Adv., 2016, 2, e1501438.

54 Y. Zhang, X. Duan, P. C. Soon, V. Sychrovsky, J. W. Canary and A. Jerschow, Chem. Phys. Chem., 2016, 17, 2967-2971.

55 Y. Zhang, P. C. Soon, A. Jerschow and J. W. Canary, Angew. Chem., Int. Ed., 2014, 53, 3396-3399.

56 S. Cavadini, J. Dittmer, S. Antonijevi and G. Bodenhausen, J. Am. Chem. Soc., 2005, 127, 15744-15748.

57 P. Ahuja, R. Sarkar, P. R. Vasos and G. Bodenhausen, J. Am. Chem. Soc., 2009, 131, 7498-7499.

58 S. J. DeVience, R. Walsworth and M. S. Rosen, NMR Biomed., 2013, 26, 1204-1212.

59 M. C. D. Tayler and M. H. Levitt, Phys. Chem. Chem. Phys., 2011, 13, 9128-9130.

60 S. Glöggler, S. J. Elliott, G. Stevanato, R. C. D. Brown and M. H. Levitt, RSC Adv., 2017, 7, 34574.

61 M. C. D. Tayler and M. H. Levitt, J. Am. Chem. Soc., 2013, 135, 2120.

62 N. S. de Groot, T. Parella, F. X. Aviles, J. Vendrell and S. Ventura, Biophys. J., 2007, 92, 1732.

63 R. W. Adams, C. M. Holroyd, J. A. Aguilar, M. Nilsson and G. A. Morris, Chem. Commun., 2013, 49, 358.

64 V. Govindaraju, K. Young and A. A. Maudsley, NMR Biomed., 2000, 13, 129.

65 G. Zheng and W. S. Price, Prog. Nucl. Magn. Reson. Spectrosc., 2010, 56, 267.

66 A. Haase, J. Frahm, W. Hanicke and D. Matthaei, Phys. Med. Biol., 1985, 30, 341.

67 M. Mescher, H. Merkle, J. Kirsch, M. Garwood and R. Gruetter, NMR Biomed., 1998, 11, 266.

68 W. R. Carper and C. E. Keller, J. Phys. Chem., 1997, 101, 3246.

69 K. Nahashima, D. Krishna Rao, G. Pagès, S. S. Velan and P. W. Kuchel, J. Biomol. NMR, 2014, 59, 31.

70 G. Pileio, J. T. Hill-Cousins, S. Mitchell, I. Kuprov, L. J. Brown, R. C. D. Brown and M. H. Levitt, J. Am. Chem. Soc., 2012, 134, 17494. 